\title{
Preparation of mNGF-conjugated Iron Oxide Nanoparticles and Repair of Peripheral Nerve Injury in Rats under Applied External Magnetic Field
}

\author{
LIJUN LI, HAIMITI ABUDUAINI, DONGKUI NI*, Y. SHI, F. ZHU AND Q. ZONG \\ Department of Orthopedics, Second Hospital Affiliated to Tianjin Medical University, Tianjin, People's Republic of China, \\ ${ }^{1}$ Department of Traumatology, Yantai Hospital, Binzhou Medical College, Yantai, People's Republic of China
}

\author{
Li, et al.: Repair of Peripheral Nerve Injury in Rats under External Magnetic Field
}

Peripheral nerve injury is a common injury that can result in various dysfunctions, such as movement and sensory disorders or permanent disabilities. Although the application of microsurgical techniques has been able to achieve accurate anastomosis at the nerve stump, the effect of nerve repair is still unsatisfactory. In recent years, the focus of research has become how to effectively improve the regeneration microenvironment after nerve injury. At present, there are several methods for the administration of exogenous neurotrophic factors, including systemic medication, local stratified puncture administration, and local application of a micro-osmotic pump. However, due to the fact that the half-life of neurotrophic factors is only 2-5 min and that these are easily inactivated in vivo, the clinical use of exogenous nerve growth factors has been limited. In the present work, using poly (lactic acid glycolic acid) copolymer as a wrapping material, conjugated mouse nerve growth factor to MNPs to prepare the magnetic mouse nerve growth factor-poly(lactic acid glycolic acid) copolymer nanoparticles by the single emulsion solvent evaporation method and its physicochemical properties were characterized. Subsequently, the drug distribution in vivo was observed by comparing magnetic resonance imaging $\mathrm{T}^{*}$ intensity values under the guidance of an external magnetic field at different time periods. Then, macroscopic, functional, electrophysiological, and histological assessments of nerves were performed at eight weeks after surgery. The results showed that the use of magnetic targeting therapy can accurately guide the aggregation of drugs, which induces the promoting of functional recovery.

Key words: Nerve growth factor, MNPs, peripheral nerve, targeting therapy, magnetic field

Peripheral nerve injury (PNI) is a common injury that can result in various dysfunctions, such as movement and sensory disorders or permanent disabilities ${ }^{[1,2]}$. Although the application of microsurgical techniques has been able to achieve accurate anastomosis at the nerve stump, the effect of nerve repair is still unsatisfactory. With the further research on the treatment of PNI, it was found that nerve regeneration and recovery of nerve function after nerve anastomosis are influenced by the microenvironment of the nerve injury. Lundborg ${ }^{[3]}$ reported the regeneration and repair of PNI and described the local microenvironment of the nerve regeneration chamber. The microenvironment consists of intact nerve channels, energetic Schwann cells, and a variety of neurotrophic factors (NFs) involved in the local blood supply. Changes in the microenvironment can affect the proliferation of Schwann cells, the release of NFs, and neurite outgrowth. In recent years, the focus of research has become how to effectively improve the regeneration microenvironment after nerve injury ${ }^{[4]}$.

NFs have been extensively studied as a restorative treatment option for peripheral neuropathies due to their role in neuronal survival, growth, and synaptic plasticity $^{[5]}$. After PNI, axons undergo Waller degeneration and Schwann cell proliferation at the stump, many kinds of NFs secrete, induce, stimulate, and regulate the regeneration of axons and the formation of the myelin sheath ${ }^{[6,7]}$. These NFs include nerve growth factor (NGF), brain-derived neurotrophic factor, glial cell-derived neurotrophic factor (GNF), and pancreatic neurotrophic factor (PNF). NGF was the first of many ontogenetic signals that was identified for the development of the nervous system ${ }^{[8]}$. It plays an indispensable role in maintaining the survival, differentiation, and maturation of sympathetic and sensory neurons and promoting axonal growth. 
At present, there are several methods for the administration of exogenous NFs, including systemic medication, local stratified puncture administration, and local application of a micro-osmotic pump. However, due to the fact that the half-life of NFs is only 2-5 min and these are easily inactivated in vivo, hence the clinical use of exogenous NGFs have been limited ${ }^{[9]}$. McCallister improved the efficacy of NGF and PNF by slowly releasing using a micro pump into the nerve endings; however, embedding the micro pump in the body can easily lead to infection and the operation is relatively complex, so it is difficult to apply in the clinic ${ }^{[10]}$.

In vitro studies have shown that conjugation to iron oxide magnetic nanoparticles (MNPs) can effectively prolong the activity of several growth factors, such as NGF and GNF ${ }^{[11,12]}$. An additional advantage of MNPs is that they can be remotely guided by a magnetic field ${ }^{[13]}$. The drug is directed to the site of injury under the guidance of an external magnetic field and released to reduce the effect on normal tissue in the nonmagnetic field. Due to multifunctional properties, such as small size effect, super paramagnetic, inherently biocompatible magnetic nanomaterial has shown an increasing number of applications in some field of medicine, such as magnetic resonance imaging $(\mathrm{MRI})^{[14,15]}$, drug delivery ${ }^{[16,17]}$, and hyperthermia, and achieved good clinical results.

In the present work, we synthesized and characterized mouse nerve growth factor (mNGF)-conjugated MNPs and the drug distribution in vivo was observed by comparing $\mathrm{MR}$ imaging $\mathrm{T} 2 *$ intensity values under the guidance of an external magnetic field at different time periods. Then, macroscopic, functional, electrophysiological and histological assessments of nerves were performed $8 \mathrm{w}$ after surgery.

\section{MATERIALS AND METHODS}

Sixty healthy Sprague Dawley (SD) rats, a combination of males and females weighing $280 \pm 10 \mathrm{~g}$, were purchased from Tianjin Experimental Animal Marketing Company. mNGF was bought from Weiminghu Bioengineering Biopharmaceutical Company, poly(lactic acid glycolic acid) copolymer (PLGA) was obtained from Germany Wins Group and the mNGF enzyme-linked immunosorbent assay (ELISA) kit was acquired from Tianjin Furuixiang Technology Company. All procedures performed in studies involving animals were in accordance with the ethical standards of the institution or practice at which the studies were conducted.

\section{Preparation of MNPs:}

Magnetic mNGF-PLGA NPs were synthesized by the single emulsion $(\mathrm{o} / \mathrm{w})$ solvent evaporation method. In brief, PLGA $(200 \mu \mathrm{g})$ was dissolved in $500 \mu \mathrm{l}$ dichloromethane solution (organic phase), then $16 \mu \mathrm{l}$ ferromagnetic iron particles were added to form a brown solution (test tube 1). We then added $18 \mu \mathrm{g}$ mNGF to $500 \mu 1$ distilled water (aqueous phase) under constant stirring (test tube 2). Test tube 1 solution was slowly added to test tube 2 in the cell disruptor. $\mathrm{O} / \mathrm{W}$ emulsion was prepared by dropwise addition of $5 \mathrm{ml} 0.5 \% \mathrm{CHA}$ solution as a stabilizing agent. After the evaporation of organic solvent, NPs collected by centrifugation at $9000 \mathrm{rpm}$ for $1 \mathrm{~h}$ at $4^{\circ}$ (cooling centrifuge, Beijing Pine Source Huaxing, China), followed by lyophilisation (Beijing Pine Source Huaxing, China).

\section{Characterization of MNPs:}

The MNP morphology was studied using transmission electron microscopy (TEM). Samples were prepared by placing one drop of a dilute suspension of MNPs in water on a carbon-coated copper grid, which was stained with phosphotungstic acid solution $(10.0 \mathrm{~g} / \mathrm{l})$ and allowed the solvents to evaporate at room temperature. The MNP particle size and distribution was studied using a laser particle sizer.

Freeze-dried nanoparticles (10 g) were dissolved in $10 \mathrm{ml}$ of acetonitrile. After achieving the suitable dilution, the weight of the MNPs was calculated with the mNGF standard curve regression equation, and entrapment efficiency (EE) and drug loading (DL) rate of the drug were calculated with the following Eqns., EE $(\%)=($ mass of drug in NPs/mass of drug added $) \times 100$ and DL $(\%)=($ drug weight in NPs/weight of NPs) $\times 100$.

The drug release behaviour of the NPs was investigated employing the ELISA method. The NPs were dispersed in $5 \mathrm{ml}$ of $\mathrm{pH} 7.4$ phosphate buffer and stored at $4^{\circ}$ until the measurements were performed over a period of $114 \mathrm{~h}$. At specific time points, 0, 0.5, 1, 2, 4, 8, 18, 28, $42,52,66,76,90$, and $114 \mathrm{~h}$, the dissolution samples $(200 \mu \mathrm{l})$ were collected and centrifuged at $9000 \mathrm{rpm}$ for $1 \mathrm{~h}$. The supernatant was subsequently placed into the ELISA plates according to the manufacturer's instructions. The resulting absorption was measured at $450 \mathrm{~nm}$ in a micro-ELISA reader. To investigate the stability of the NPs during the in vitro release study, 
the released amount of magnetic mNGF-PLGA NPs was calculated from the mNGF standard curve and a cumulative release curve was plotted.

\section{Preparation of rat sciatic nerve model:}

The animals were anesthetized by intraperitoneal injection of $10 \%$ chloral hydrate $(0.30 \mathrm{ml} / 100 \mathrm{~g})$. After satisfactory anaesthesia, fur in the surgical area was shaved and the animal was fixed in the prone position. The operative area was thoroughly disinfected, the sciatic nerve in the left hind limb was exposed and at a site approximately $1 \mathrm{~cm}$ apart from the lower edge of the piriformis muscle, the sciatic nerve was transversely cut. At the proximal and distal ends of the nerve, the outer membrane was loop stripped the outer membrane and the $1 \mathrm{~mm}$ long nerve bundle was excised. Eversion suture of anastomotic stoma and inter-line breakage was performed with 9-0 non-traumatic sutures, resulting in an approximately $2.0 \mathrm{~mm}$ nerve defect. At the same time, the lateral and proximal sides of the anastomosis were sutured with 8-0 soft tissue sutures to reduce the tension of the anastomosis.

\section{MRI:}

According to the fact that as contrast agents for $\mathrm{MRI}^{[18]}$, MNPs can shorten the T2* signals, providing darker colour and decreasing $\mathrm{T} 2 *$ values as compared to unlabelled tissues ${ }^{[19,20]}$. After injuring the left sciatic nerve in the rats, we used T2* MRI (Philips Achieva $3.0 \mathrm{~T}$, TR $6369 \mathrm{~ms}$, TE $108 \mathrm{~ms}$ ) to scan the left and right lower limbs at different time periods (before the injection of drugs, after the injection of drugs, and when the left lower limb was fixed on an external magnetic field for $2 \mathrm{~h}$ ). Drug distribution in vivo was observed by comparing T2* values.

\section{Functional and electrophysiological evaluations:}

For the experiments to evaluate the functional recovery of the sciatic nerve, 60 rats were divided into three groups, group $\mathrm{A}$, which received an intravenous injection of $2 \mathrm{ml}$ magnetic mNGF-PLGA NP suspension+left lower limb fixed on an external magnetic field (1T) for $2 \mathrm{~h}$ per week; group B, which only received an intravenous injection of $2 \mathrm{ml}$ magnetic mNGF-PLGA NP suspension weekly and group C, which received a weekly intravenous injection of $2 \mathrm{ml}$ mNGF water solution.

To evaluate the functional recovery of the injured nerve, a walking track analysis was performed as described in reference ${ }^{[21]}$. At $8 \mathrm{w}$ after the surgery, the sciatic functional index (SFI) was estimated from a walking track test ${ }^{[22,23]}$. Briefly, the animals walked down on the floor paper impregnated with carbon ink and we obtained the corresponding footprints. The distance between the first and fifth toes (TS), the distance between the second and fourth toes (IT), and the print length (PL) were measured. The SFI was calculated according to the Eqn., SFI=109.5 (ETSNTS)/NTS-38.3 (EPL-NPL)/NPL+13.3(EIT-NIT)/ $\mathrm{NIT}-8.8$. SFI $=0$ represents the normal nerve function and $\mathrm{SFI}=100$ represents complete dysfunction.

Functional outcome of nerve regeneration was measured electrophysiologically on the left sciatic nerve at eight weeks after surgery using the MEB-7102 instrument (Nihon Koden, Osaka, Japan). Under anaesthesia, the left sciatic nerve was exposed and the proximal and distal regenerated nerves were stimulated. The compound muscle action potentials were measured and the velocity of the nerve conduction was calculated.

The rats were sacrificed, the bilateral sides of the triceps surae were excised, the surface blood was washed with physiological saline, dried on a filter paper, and weighed and recorded their weights with an electronic balance scale (BS124S, Sartorius AG, Germany). The wet weight recovery ratio=the weight of the triceps surea of the operational side/the weight of the triceps surae of the non-operational side.

\section{Histological analysis:}

At eight weeks postoperatively, the sciatic nerves were obtained from each group and fixed in $4 \%$ paraformaldehyde. After fixation, the samples were washed with PBS, dehydrated in a graded sucrose solution series, cleared in xylene, and embedded in paraffin. The samples were then cut into 5 - $\mu \mathrm{m}$ sections for hematoxylin and eosin (HE) staining and observed on a microscope.

\section{Statistical analysis:}

All of the results are reported as mean \pm SD. Statistical comparisons among groups involved the use of the SPSS 13.0 software (SPSS, Chicago, Ill., USA). Statistical significance was ascribed to the data when $\mathrm{p}<0.05$.

\section{RESULTS AND DISCUSSION}

Magnetic mNGF-conjugated NPs were synthesized using the single emulsion $(\mathrm{o} / \mathrm{w})$ solvent evaporation method, which appeared as brown suspension with no 
delamination (fig. 1). The particle size and distribution of the MNPs was observed under a laser particle sizer. MNPs were relatively uniform in size (fig. 2) and the mean size of the MNPs was $205.9 \pm 8.63 \mathrm{~nm}$. The morphology and structure of the particles were observed by TEM, and the images indicate that the particles are quasi-spherical and contain numerous well-dispersed magnetic iron particles (fig. 1). The calculated drug-loading rate and the encapsulation efficiency of the PLGA microspheres were 7.40 and $84.61 \%$, respectively.

As shown in fig. 3, burst release of magnetic mNGFconjugated MNPs was evident with approximately $30 \%$ of mNGF was released within the initial $12 \mathrm{~h}$ and the drug release was slow and continued till the d 5. The initial burst release of mNGF was probably due to the drug, which was adsorbed or associated with the surface of the nanoparticles. The colour changes can be seen in fig. 3; with the increase of release concentration, the colour of would turn from the initial white to pale brown.

After the rat left sciatic nerve injury model was established, the in vivo aggregation of the drug was

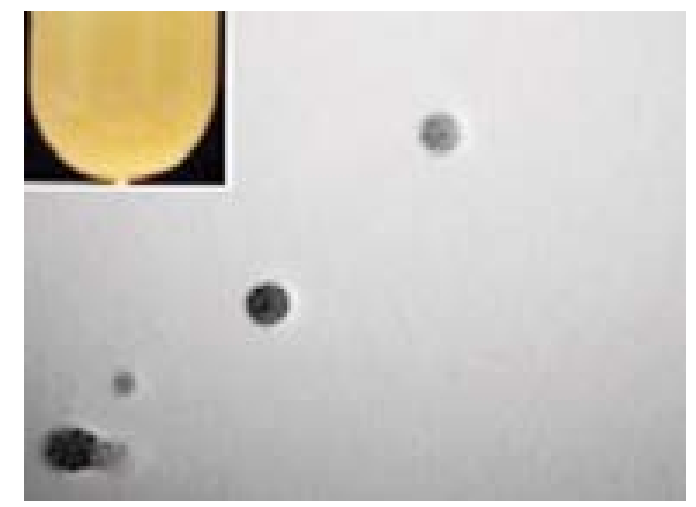

Fig. 1: TEM images and appearance of magnetic mNGF-PLGA nanoparticles

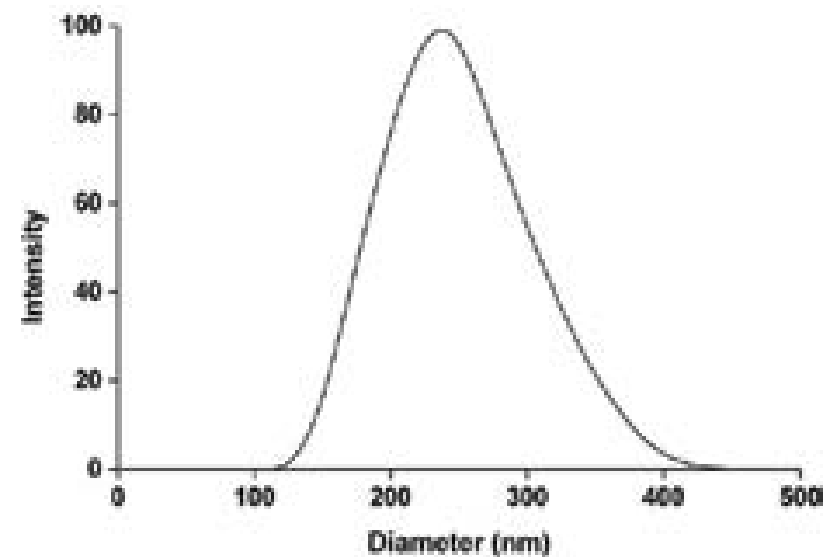

Fig. 2: Size distribution
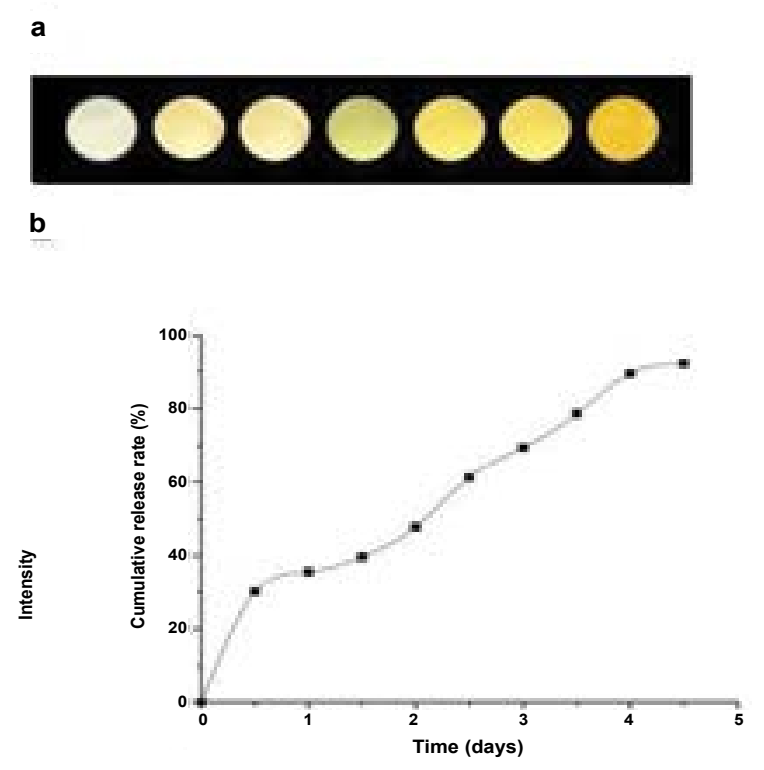

Fig. 3: (a) Colour changes of ELISA kit well and (b) cumulative release curve in vitro

(一- Magnetic nanoparticles release in in vitro

observed under the MRI. As shown in fig. 4a, the left and right lower limb T2* signals were high in group 1 (before the injection of the drug), while the T2* intensity values were 312.68 and 314.74 , respectively. In group 2 (after the injection of the drug), T2* signals were decreased; however, T2* intensity values of the left and right lower limbs were 264.43 and 263.78, respectively. The left lower limb $\mathrm{T} 2 *$ signals were significantly weakened in group 3 ( $2 \mathrm{~h}$ fixation of the left lower limb in the external magnetic field); the T2* intensity value was 150.90 , which was much lower than the right lower limb, 233.54. The contrast histogram of the left and right lower limbs of the rats (fig. 4b), demonstrating that there was a significant difference of T2* intensity values of the left and right lower limbs in group $3(p<0.05)$ and there was no significant difference in groups 1 and 2 . This indicated that the magnetic mNGF-PLGA NPs aggregated well under the external magnetic field.

Preoperatively, the SFI in the rats in all groups, group A, groupBand group $\mathrm{C}$, were approximatelyzero, indicating the normal function. After injury, the SFI decreased to approximately -100 , indicating the complete loss of function. Eight weeks after the surgery, the SFI was calculated according to the corresponding footprints (figs. 5a, b and c). As shown in fig. 6, in group A, the SFI was $(-34.91 \pm 7.86)$; in group $\mathrm{B}$, the SFI was $(-56.97 \pm 10.29)$ and in group $C$, it was $(-61.68 \pm 9.56)$. The recovery of SFI in group A showed a significant 
a

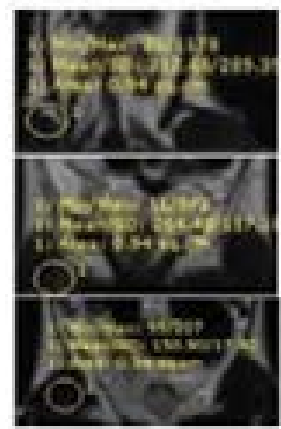

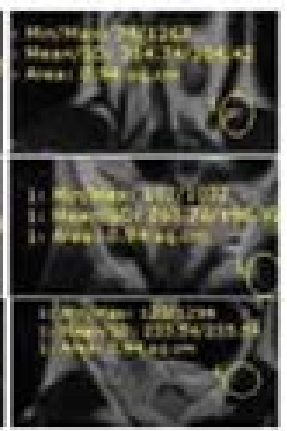

b

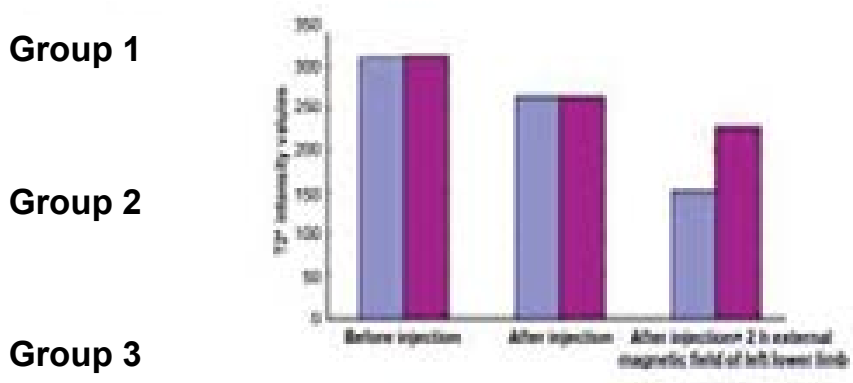

Fig. 4: Comparative T2* intensity and contrast histograms of the rat left and right lower limbs

(a) Comparison of $\mathrm{T} 2$ * intensity values, (b) contrast histogram of the left and right lower limbs of the rats demonstrating that there was a significant difference in $\mathrm{T} 2$ \% intensity values of the left and right lower limbs in group $3(\mathrm{p}<0.05)$ and there was no significant difference in groups 1 and 2 . Blue colour indicates left lower limb, pink colour indicates right lower limb

a

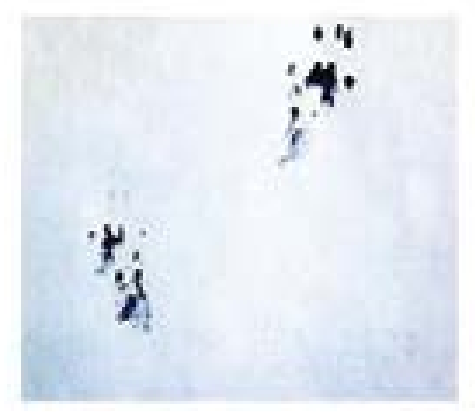

b

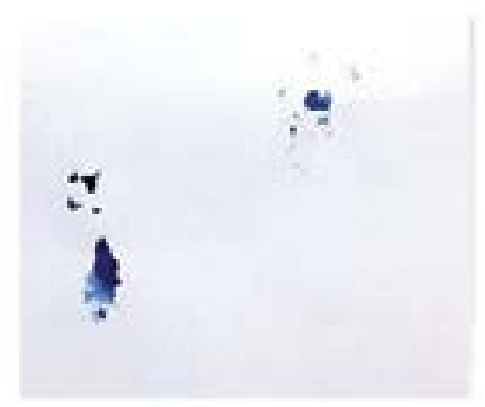

c

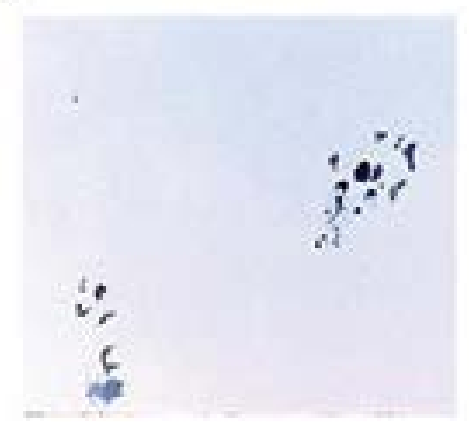

Fig. 5: Footprint analysis

Footprint analysis of (a) group A (intravenous injection of $2 \mathrm{ml}$ magnetic mNGF-PLGA NP suspension left lower limb fixed on external magnetic field, (b) group B (intravenous injection of $2 \mathrm{ml}$ magnetic mNGF-PLGA nanoparticles suspension) and (c) group $\mathrm{C}$ (intravenous injection of $\mathbf{2} \mathbf{~} \mathrm{m} \mathbf{m N G}$ water solution)

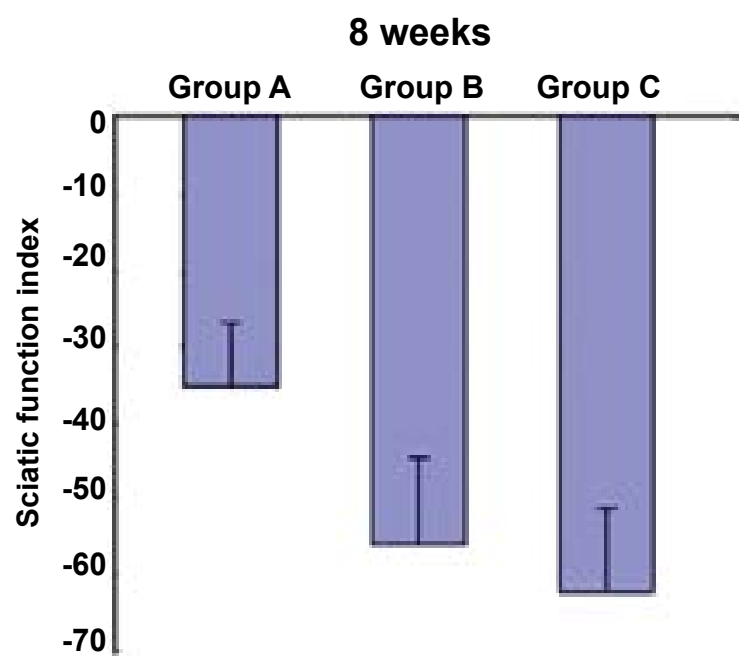

Fig. 6: Mean function recovery of each group after sciatic nerve transection and repair

Comparison of the mean function recovery of each group after sciatic nerve transection and repair through functional analysis of neural regeneration at $8 \mathrm{w}$ after surgery using walking track analysis. Measurements made from walking track prints were submitted to an SFI difference compared with groups $\mathrm{B}$ and $\mathrm{C}(\mathrm{p}<0.05)$, and there was no significant difference between groups $\mathrm{B}$ and $\mathrm{C}$.

The motor nerve conduction velocity (MCV) measurements at $8 \mathrm{w}$ after surgery are listed in fig. 7. The MCV was $31.00 \pm 3.30 \mathrm{~m} / \mathrm{s}$ for group A, which was greater than $21.30 \pm 4.79 \mathrm{~m} / \mathrm{s}$ for group $\mathrm{B}$, and $23.15 \pm 4.53 \mathrm{~m} / \mathrm{s}$ for group C. Group A was significantly different from the other groups $(\mathrm{p}<0.05)$, and there was no significant difference between groups $\mathrm{B}$ and $\mathrm{C}$.

Eight weeks after surgery, the triceps surae of the injured side had different degrees of atrophy and the recovery ratio of muscle wet weight in group A was the highest $(0.51+0.09)$ as groups $\mathrm{B}$ and $\mathrm{C}$ were $(0.41 \pm 0.06)$ and $(0.39 \pm 0.06)$, respectively. Group A was significantly different from the other groups $(\mathrm{p}<0.05)$, and there was no significant difference between groups $\mathrm{B}$ and $\mathrm{C}$.

Eight weeks after surgery, the injured sciatic nerve tissues were extracted and observed under a microscope. 
As shown in fig. 8, the most injured sciatic nerves were observed in group A. A large number of regenerated nerve fibres were seen, which demonstrated the most significantly promoted recovery (fig. 8a). Group B samples showed significantly promoted recovery of injured nerves, and more regular nerve fibres can be observed (fig. 8b). Group C samples showed irregular nerve fibres and there was a lot of scar tissue between the ends (fig. $8 \mathrm{c}$ ).

Peripheral nerve regeneration is still a challenge in the clinic. NGF is often viewed as the prototype for all neurotrophins and considered to be a trophic molecule that is critical for the survival of sympathetic and sensory neurons ${ }^{[24]}$. However, growth factors have limited effectiveness when applied externally due to a short biological half-life and rapid degradation in $v i v o^{[25,26]}$. Here, we described an approach to increase the stability and aggregation of mNGF using MNPs under the guidance of external magnetic field.

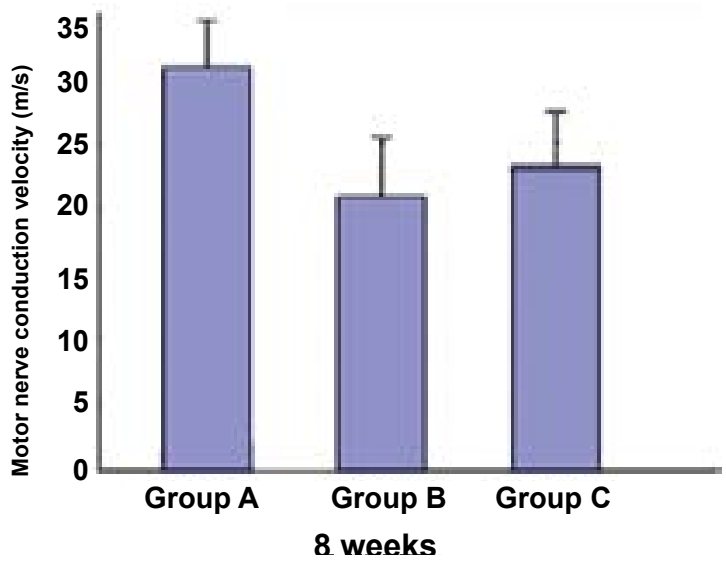

Fig. 7: Comparison of the MCV at 8 weeks after surgery Motor nerve conduction velocity (MCV) was significantly higher in group A compared to that of groups $B$ and $C(p<0.05)$ and there was no significant difference between groups $B$ and C. Data expressed as mean \pm SEM
The emulsification solvent evaporation method has attracted increasing interest in biomedical and clinical research. It has been applied in the field of drug delivery, tissue engineering, and $\mathrm{MRI}^{[27,28]}$. The advantage of this technique is that the particles are of small size, the proper size range with a broad distribution, relatively high magnetization, and good spherical morphology and they have a simplified preparation process ${ }^{[29]}$. The release rate of the particles was determined in vitro, and the release rate of $\mathrm{mNGF}$ reached $92 \%$ on the $\mathrm{d} 5$. PLGA wrapped in $\mathrm{mNGF}$ as a coating material plays a protective role in NGF to avoid premature inactivation in the external environment. PLGA nanoparticles have certain advantages, such as the probability to prolong drug release, control drug release, decrease drug degradation, and increase drug bioavailability ${ }^{[30,31]}$. Drugs were injected and the MR imaging T2* intensity values of the left and right lower limbs in each group were compared to confirm the aggregation of the drugs in vivo. The results showed that the magnetic mNGFPLGA NPs have promising aggregation in vivo under the external magnetic field.

A functional evaluation was preferred to determine the effectiveness of the NGF treatment in each group. The SFI provides general information about nerve regeneration and functional recovery because it represents the outcome of the repair process. It has been proven to be reliable, repeatable, and highly useful for determining motor function following compression and stretch injury ${ }^{[32]}$, nerve graft or conduit ${ }^{[33]}$, and surgical repair ${ }^{[34]}$. The degree of recovery of sciatic nerve function was assessed by measuring the SFI. The data obtained showed that at $8 \mathrm{w}$ after surgery, an increasing ability to walk became evident. A better and faster functional recovery was shown in group A. In contrast, the functional recovery progressed more a

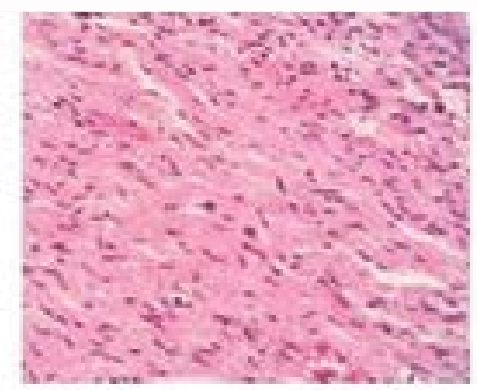

b

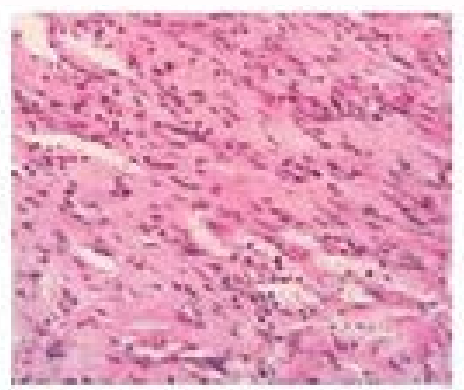

c

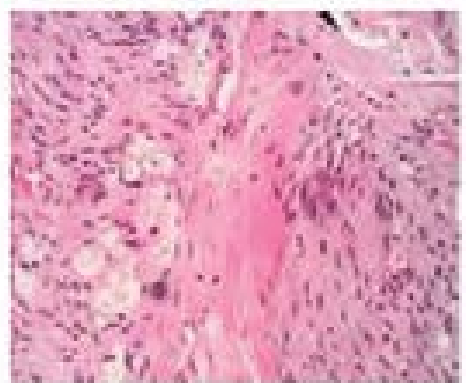

Fig. 8: Histological analysis

Histological analysis of (a) HE staining of the sciatic nerves in group A rats ((intravenous injection of 2 ml magnetic mNGF-PLGA NP suspension left lower limb fixed on external magnetic field), (b) the gap between the ends of the nerve fibers in group B rats (intravenous injection of $2 \mathrm{ml}$ magnetic mNGF-PLGA nanoparticles suspension) and (c) HE staining of the sciatic nerves in group $\mathrm{C}$ rats (intravenous injection of $2 \mathrm{ml} \mathbf{m N G F}$ water solution) 
slowly in group C. The recovery of the group A was superior, indicating that NGF has the effect of promoting functional recovery under the guidance of an external magnetic field.

In addition, to further understand the specific process during functional recovery, MCV was evaluated next. In this study, the MCV of the regenerating nerves was faster in group A than in the other groups at $8 \mathrm{w}$ (fig. 7). There were significant differences between group A than groups B and $\mathrm{C}$. These findings indicate that the administration of mNGF significantly inhibited the decrease of MCV and promoted functional recovery following sciatic nerve transection in adult rats. Finally, the histologic studies supported (fig. 8), the functional recovery data obtained from these tests. The promotion of SFI and MCV recovery by the treatment of mNGF also confirmed these findings.

In conclusion, the data from the present study show that the use of magnetic targeting therapy can accurately guide the aggregation of drugs, which induces the promoting of functional recovery, and enhance nerve regeneration of the sciatic nerves in rats. At present, magnetic mNGF-conjugated NPs have not been used in the clinical treatment of PNI. Therefore, in future work, the research results would be combined with practical applications to provide an effective and feasible treatment method for PNI.

\section{Acknowledgements:}

This work was supported by a grant from Tianjin Municipal Commission of Health and Family Planning (GM 2015KZ094). The authors would like to thank for Prof. Xiaoqun Gong excellent technical support and critically reviewing the manuscript.

\section{Conflict of interest:}

The all authors report no conflicts of interest in this work.

\section{REFERENCES}

1. Faglioni W, Siqueira MG, Martins RS, Heise CO, Foroni L. The epidemiology of adult traumatic brachial plexus lesions in a large metropolis. Acta Neurochir (Wien) 2014;156:1025-8.

2. Martins RS, Bastos D, Siqueira MG, Heise CO, Teixeira MJ. Traumatic injuries of peripheral nerves: a review with emphasis on surgical indication. Arq Neuropsiquiatr 2013;71:811-4.

3. Lundborg G. Nerve regeneration and repair. A review. Acta Orthop Scand 1987;58:145-69.

4. Webber $\mathrm{C}$, Zochodne $\mathrm{D}$. The nerve regenerative microenvironment: early behavior and partnership of axons and Schwann cells. Exp Neurol 2010;223:51-9.
5. Goss JR. The therapeutic potential of gene transfer for the treatment of peripheral neuropathies. Expert Rev Mol Med 2007;9:1-20.

6. Hontanilla B, Aubá C, Gorría O. Nerve regeneration through nerve autografts after local administration of brain-derived neurotrophic factor with osmotic pumps. Neurosurgery 2007;61:1268-74.

7. Gravvanis AI, Tsoutsos DA, Tagaris GA, Papalois AE, Patralexis CG, Iconomou TG, et al. Beneficial effect of nerve growth factor-7S on peripheral nerve regeneration through inside-out vein grafts: an experimental study. Microsurgery 2004;24:408-15.

8. Levi-Montalcini R. The nerve growth factor 35 years later. Science 1987;237:1154-62.

9. Pradat PF. Treatment of peripheral neuropathies with neutrotrophic factors: animal models and clinical trials. Rev Neurol 2003;159:147-61

10. McCallister WV, Tang P, Smith J, Trumble TE. Axonal regeneration stimulated by the combination of nerve growth factor and ciliary neurotrophic factor in an end-to-side model. J Hand Surg Am 2001;26:478-88.

11. Zhang S, Uludag H. Nanoparticulate systems for growth factor delivery. Pharm Res 2009;26:1561-80.

12. Ziv-Polat O, Shahar A, Levy I, Skaat H, Neuman S, Fregnan $\mathrm{F}$, et al. The role of neurotrophic factors conjugated to iron oxide nanoparticles in peripheral nerve regeneration: in vitro studies. BioMed Res Int 2014;2014:1-10.

13. Giannaccini M, Calatayud MP, Poggetti A, Corbianco S, Novelli M, Paoli M, et al. Magnetic nanoparticles for efficient delivery of growth factors: stimulation of peripheral nerve regeneration. Adv Healthc Mater 2017;6:1601429.

14. Veiseh O, Sun C, Gunn J, Kohler N, Gabikian P, Lee D, et al. Optical and MRI Multifunctional Nanoprobe for Targeting Gliomas. Nano Lett 2005;5:1003-8.

15. Jun YW, Huh YM, Choi JS, Lee JH, Song HT, Kim S, et al. Nanoscale size effect of magnetic nanocrystals and their utilization for cancer diagnosis via magnetic resonance imaging. Am Chem Soc 2005;127:5732-3.

16. Alexiou C, Arnold W, Klein RJ, Parak FG, Hulin P, Bergemann $\mathrm{C}$, et al. Locoregional cancer treatment with magnetic drug targeting. Cancer Res 2000;60:6641-8.

17. Song M, Zhang R, Dai Y, Gao F, Chi H, Lv G, et al. The in vitro inhibition of multidrug resistance by combined nanoparticulate titanium dioxide and UV irradiation. Biomaterials 2006;27:4230-8.

18. Mejías R, Pérez-Yagüe S, Gutiérrez L, Cabrera LI, Spada R, Acedo $\mathrm{P}$, et al. Dimercaptosuccinic acid-coated magnetite nanoparticles for magnetically guided in vivo delivery of interferon gamma for cancer immunotherapy. Biomaterials 2011;32:2938-52.

19. Wang YX, Hussain SM, Krestin GP. Superparamagnetic iron oxide contrast agents: physicochemical characteristics and applications in MR imaging. Eur Radiol 2001;11:2319-31.

20. Xu P, Shen Z, Zhang B, Wang J, Wu R. Synthesis and characterization of superparamagnetic iron oxide nanoparticles as calcium-responsive MRI contrast agents. Appl Surf Sci 2016;389:560-6.

21. Zhu S, Ge J, Wang Y, Qi F, Ma T, Wang M, et al. A synthetic oxygen carrier-olfactory ensheathing cell composition system for the promotion of sciatic nerve regeneration. Biomaterials 2014;35:1450-61. 
22. Yin ZS, Zhang H, Gao W. Erythropoietin promotes functional recovery and enhances nerve regeneration after peripheral nerve injury in rats. AJNR Am J Neuroradiol 2010;31:509-15.

23. Zhang W, Gao Y, Zhou Y, Liu J, Zhang L, Long A, et al. Tang Localized and sustained delivery of erythropoietin from PLGA microspheres promotes functional recovery and nerve regeneration in peripheral nerve injury. BioMed Res Int 2015;2015:478103.

24. Sun W, Sun C, Zhao H, Lin H, Han Q, Wang J, et al. Improvement of sciatic nerve regeneration using lamininbinding human NGF-beta. PLoS One 2009;4:e6180.

25. Zhang YZ, Moheban DB, Conway BR, Bhattacharyya A, Segal RA. Cell surface Trk receptors mediate NGF-induced survival while internalized receptors regulate NGF-induced differentiation. J Neurosci 2000;20:5671-8.

26. Tuszynski MH, Thal L, Pay M. A phase 1 clinical trial of nerve growth factor gene therapy for Alzheimer disease. Nat Med. 2005;11(5):551-555.

27. Rosengart AJ, Kaminski MD, Chen H, Caviness PL, Ebner $\mathrm{AD}$, Ritter JA. Magnetizable implants and functionalized magnetic carriers: a novel approach for noninvasive yet targeted drug delivery. J Magn Magn Mater 2005;293:633-8.

28. Kaminski MD, Rosengart AJ. Detoxification of blood using injectable magnetic nanospheres: A conceptual technology description. J Magn Magn Mater. 2005;29:398-403.

29. Liu X, Kaminski MD, Riffle JS, Chen H, Torno M, Finck $\mathrm{MR}$, et al. Preparation and characterization of biodegradable magnetic carriers by single emulsion-solvent evaporation. J Magn Magn Mater 2007;311:84-7.
30. Pillai GJ, Paul-Prasanth B, Nair SV, Menon D. Influence of surface passivation of 2- methoxyestradiol loaded PLGA nanoparticles on cellular interactions, pharmacokinetics and tumour accumulation. Colloids Surf B Biointerfaces 2017;150:242-9.

31. Takeuchi I, Fukuda K, Kobayashi S, Makino K. Transdermal delivery of estradiol-loaded PLGA nanoparticles using iontophoresis for treatment of osteoporosis. Biomed Mater Eng 2016;27:475-83.

32. Spiege DA, Seaber AV, Chen LE, Urbaniak JR. Recovery following stretch injury to the sciatic nerve of the rat: an in vivo study. J Reconstr Microsur 1993;9:69-74.

33. Chen LE, Seaber AV, Urbaniak JR, Murrell GA. Denatured muscle as a nerve conduit: a functional, morphologic, and electrophysiologic evaluation. J Reconstr Microsurg 1994;10:137-44.

34. de Medinaceli L, Seaber AV. Experimental nerve reconnection: importance of initial repair. Microsurgery 1989;10:56-70.

This is an open access article distributed under the terms of the Creative Commons Attribution-NonCommercial-ShareAlike 3.0 License, which allows others to remix, tweak, and build upon the work non-commercially, as long as the author is credited and the new creations are licensed under the identical terms

This article was originally published in a special issue, "XXXXXX"

Indian J Pharm Sci 2020:82(1)spl issue1; xx-xx 